\title{
Database Normalization as a By-product of Minimum Message Length Inference
}

\author{
David L. Dowe and Nayyar Abbas Zaidi \\ Clayton School of I.T., Monash University, Clayton, Vic. 3800, Australia \\ \{david.dowe, nayyar.zaidi\}@infotech.monash.edu.au
}

\begin{abstract}
Database normalization is a central part of database design in which we re-organise the data stored so as to progressively ensure that as few anomalies occur as possible upon insertions, deletions and/or modifications. Successive normalizations of a database to higher normal forms continue to reduce the potential for such anomalies. We show here that database normalization follows as a consequence (or special case, or byproduct) of the Minimum Message Length (MML) principle of machine learning and inductive inference. In other words, someone (previously) oblivious to database normalization but well-versed in MML could examine a database and - using MML considerations alone - normalise it, and even discover the notion of attribute inheritance.
\end{abstract}

Keywords: Minimum Message Length, MML, Database Normalization, Machine Learning, Data Mining, Intelligent Databases.

\section{Introduction}

The table is a basic building block of a Relational Database Management System (RDBMS) [1, 2]. Consequently, the structure of one or more tables in the database is of great interest. Typically, the information is structured into tables during the Entity-Relationship (ER) diagram phase of conceptual database design. Database normalization [3] is a process of evaluating and correcting table structures to minimize data redundancies, thereby reducing the likelihood of data anomalies upon insertion (adding a new row), deletion (deleting a row) or modification (modifying a row). Normalization is typically the heart of any database design activity.

Database normalization [2] works through a series of stages called normal forms (NFs) (described in sec. 3). A primary reason is to minimize data redundancies and get rid of update, delete and insertion anomalies. Also, designers would like to apply the 'minimal data rule' to the structure, making sure that all information has been captured and every piece of information captured is meaningful. In short, after the initial design is complete in the form of an Entity-Relationship (ER) diagram, designers generally analyze the relationships that exist among attributes within entities through normalization and improve the structure if need arises. 
As described, there can be many motivations behind a database normalization. In this paper, we present a novel information-theoretic perspective of database normalization. We consider the structure of the table(s) as a modelling problem for Minimum Message Length (MML) (see sec. 2). MML seeks a model giving the shortest two-part coding of model and data. If we consider table structure as a model which encodes data, MML advocates that we should be particularly interested in the variation of the encoding length of model and data as the normalization process re-structures tables for efficient design. We will consider a simple example and apply normalization to illustrate our point. As we will see in sec.4. normalization into higher forms minimizes code length (or message length) by re-structuring the relational tables. Hence, if we apply the MML principle to a relational database, then - provided we have sufficient data - we are likely to get our database normalized.

The rest of the paper is organized as follows: we introduce the minimum message length (MML) framework in sec. 2. In sec. 3. we explain a typical normalization procedure with an example. We explain the MML view of normalization in sec. 4. We conclude in sec. 5

\section{Minimum Message Length}

Minimum message length (MML), introduced by Wallace and Boulton [4] , is an important stream of studying the complexity of a data set [4-10]. It is based on Shannon's theory of information and equivalently on the theory of Turing machines and Kolmogorov complexity [6, 7, 10]. MML considers any given string $S$ as being a representation in some (unknown) code about the real world. It seeks a ([concatenated] two-part) string $I=H: A$ where the first part $H$ specifies (or encodes) a hypothesis about the data $S$ and the second part $A$ is an encoding of the data using the encoded hypothesis. If the code or hypothesis is true, the encoding is efficient (like Huffman or arithmetic codes). According to Shannon's theory, the length of the string coding an event $E$ in an optimally efficient code is given by $-\log _{2}(\operatorname{Prob}(E))$, so the length of $A$ is given by:

$$
\# A=-\log _{2}(f(S \mid H))
$$

where $f(S \mid H)$ is the conditional probability (or statistical likelihood) of data $S$ given the hypothesis $H$. Using an optimal code for specification, the length \#H of the first part of the MML message is given by $-\log _{2}(h(H))$, where $h(\cdot)$ is the prior probability distribution over the set of possible hypotheses. Using equation (11), the total two-part message length \#I is:

$$
\begin{aligned}
\# I & =\# H+\# A=-\log _{2}(h(H))-\log _{2}(f(S \mid H)) \\
& =-\log _{2}(h(H) \times f(S \mid H))
\end{aligned}
$$

The minimization of $\# I$ is equivalent to the maximization of $h(H) \times f(S \mid H)=$ $\operatorname{Prob}(H, S)$, that is the joint probability of hypothesis and data. It is thus formally equivalent to choosing the hypothesis $H$ of highest Bayesian posterior probability (not a density) given $S[11$, secs. 2 and 6.1] [8, sec. 11.3.1] 9 , footnote 158]. 


\section{A Typical Normalization Procedure - An Example}

As hinted in sec. 1, normalization works through a series of stages called normal forms. The first three stages are called first normal form (1NF), second normal form $(2 \mathrm{NF})$ and third normal form (3NF). From a structural point of view, $2 \mathrm{NF}$ is better than $1 \mathrm{NF}$, and $3 \mathrm{NF}$ is in turn better than $2 \mathrm{NF}$. For at least many purposes, $3 \mathrm{NF}$ is sufficient. A properly designed $3 \mathrm{NF}$ structure can also meet the requirements of higher normal forms - such as, e.g., Boyce-Codd Normal Form (BCNF), fourth normal form (4NF) or fifth normal form (5NF).

As an example, suppose we wish to create a database of university student enrolments. Let us assume that a student can take more than one unit, perhaps as many units as she likes. A student can also take the same unit more than once but not in one semester. Each student has a supervisor (denoted as 'lecturer' in the following discussion). A supervisor can have more than one student, but a student can only have one supervisor. Based on this information, we can proceed with the normalization procedure as follows, initially using this small example of $L=11$ rows in $1 \mathrm{NF}$ :

1NF: The term 1NF describes a tabular data format where the following properties hold. First, all of the key attributes are defined. Second, there are no repeating groups in the table -i.e., in other words, each row/column intersection (or cell) contains one and only one value, not a set of values. Third, all attributes are dependent on the primary key (PK). Based on the information given to us, we can structure data in the table Student-Rec and can use Stud-ID, Unit-No and Yr-Sem attributes as parts of the PK. The table in 1NF is shown in table1,

2NF: A table is in 2NF if the following conditions hold. First, it is in 1NF. Second, it includes no partial dependencies, that is no attribute is dependent on

Table 1. Student-Rec in 1NF. PK = ( $\underline{\text { Stud-ID }}, \underline{\text { Unit-No }}, \underline{\text { Yr-Sem }})$

\begin{tabular}{|c|c|c|c|c|c|c|c|c|c|}
\hline Stud-ID & Stud-Name & Stud & Stud- & Unit-No & Unit-Name & Lect-No & Lect-Name & Yr-Sem & Gra \\
\hline 212 & Bob Smith & Notting Hill & MIT & $\overline{\text { FIT2014 }}$ & Database Design & 47 & Geoff Yu & 2007 & $\bar{D}$ \\
\hline 212 & Bob Smith & Notting Hill & MIT & EE 1007 & Circuit Design & 47 & Geoff $\mathrm{Yu}$ & 2006 & $\mathrm{P}$ \\
\hline 213 & John News & Caufield & $\mathrm{BSc}$ & FIT3014 & Algorithm Theory & 122 & June Matt & 2007 & HD \\
\hline 214 & Alice Neal & Clayton S & $\mathrm{BSc}$ & FIT2014 & Database Design & 122 & June Matt & 2007 & HD \\
\hline 214 & Alice Neal & Clayton S & $\mathrm{BSc}$ & FIT3014 & Algorithm Theory & 122 & June Matt & 2007 & $\mathrm{D}$ \\
\hline 215 & Jill Wong & Caufield & MIT & FIT2014 & Database Design & 47 & Geoff $\mathrm{Yu}$ & 2007 & D \\
\hline 216 & Ben $\mathrm{Ng}$ & Notting Hill & BA & MT2110 & Mathematics-II & 47 & June Matt & 2007 & D \\
\hline
\end{tabular}

Table 2. Student in $2 \mathrm{NF} . \mathrm{PK}=\underline{\text { Stud-ID }}$

\begin{tabular}{|cccccc|}
\hline Stud-ID & Stud-Name & Stud-Address & Stud-Course & Lect-No & Lect-Name \\
\hline \hline 212 & Bob Smith & Notting Hill & MIT & 47 & Geoff Yu \\
213 & John News & Caufield & BSc & 122 & June Matt \\
214 & Alice Neal & Clayton S & BSc & 47 & Geoff Yu \\
215 & Jill Wong & Caufield & MIT & 47 & Geoff Yu \\
216 & Ben Ng & Notting Hill & BA & 122 & June Matt \\
\hline
\end{tabular}


Table 3. Unit in $2 \mathrm{NF}$ and $3 \mathrm{NF}, \mathrm{PK}=\underline{\text { Unit-No }}$

\begin{tabular}{|cc|}
\hline Unit-No & Unit-Name \\
\hline FIT2014 & Database Design \\
FIT3014 & Algorithm Theory \\
EE1007 & Circuit Design \\
MT2110 & Mathematics-II \\
\hline
\end{tabular}

Table 4. Stu-Unit-Rec in $2 \mathrm{NF}$ and $3 \mathrm{NF} . \mathrm{PK}=(\underline{\text { Stud-ID }}, \underline{\text { Unit-No}}, \underline{\text { Yr-Sem }})$

\begin{tabular}{|cccc|}
\hline Stud-ID & Unit-No & Yr-Sem & Grade \\
\hline 212 & FIT2014 & 2007 & D \\
212 & FIT3014 & 2007 & HD \\
212 & EE1007 & 2006 & P \\
213 & FIT 3014 & 2007 & HD \\
213 & EE1007 & 2007 & HD \\
214 & FIT2014 & 2007 & HD \\
214 & FIT3014 & 2007 & D \\
215 & FIT 2014 & 2007 & D \\
215 & FIT2014 & 2008 & D \\
216 & EE1007 & 2007 & P \\
216 & MT2110 & 2007 & D \\
\hline
\end{tabular}

Table 5. Student in $3 N F . P K=\underline{\text { Stud-ID }}$

\begin{tabular}{|ccccc|}
\hline Stud-ID & Stud-Name & Stud-Address & Stud-Course & Lect-No \\
\hline \hline 212 & Bob Smith & Notting Hill & MIT & 47 \\
213 & John News & Caufield & BSc & 122 \\
214 & Alice Neal & Clayton S & BSc & 47 \\
215 & Jill Wong & Caufield & MIT & 47 \\
216 & Ben Ng & Notting Hill & BA & 122 \\
\hline
\end{tabular}

only a portion of the primary key. The table Student-Rec in table 1 has partial dependencies. It is clear that Unit-Name only depends on Unit-No and not on the whole PK - that is, (Stud-ID, Unit-No,Yr-Sem). Also Name, Address, Course, Lect-No and Lect-Name depend only on Stud-ID. To structure the tables in 2NF, we need to eliminate these partial dependencies. A proposed design modification is shown in tables 2, 3 and 4. Table 1 is split into three tables: Student, Unit and Stu-Unit-Rec. Note that there are no partial dependencies in all of these three tables. In each table, each non-key attribute depends on all attributes in the PK. For our example, the 2NF tables are tables 2 , 3 and 4 .

3NF: A table is in 3NF if the following holds. First, it is in 2NF. Second, it contains no transitive dependencies. A transitive dependency exists when there are functional dependencies 1 such that $X \rightarrow Y, Y \rightarrow Z$ and $X$ is the primary key attribute. Looking at our design in $2 \mathrm{NF}$, there exists a transitive dependency in the Student table (table 2), where Stud-ID $\rightarrow$ Lect-No $\rightarrow$ Lect-Name. This transitive dependency can be eliminated by breaking the Student table into the Student and Lecturer tables as shown in tables [5] and 6. Note the Unit and StuUnit-Rec tables in tables 3 and 4 are already in $3 \mathrm{NF}$. For our example, the $3 \mathrm{NF}$ tables are tables 3, 4, 5, and 6.

\footnotetext{
${ }^{1}$ The attribute $B$ is fully functional dependent on the attribute $A$ if each value of $A$ determines one and only value of $B$.
} 
Table 6. Lecturer in $3 \mathrm{NF}, \mathrm{PK}=\underline{\text { Lect-No }}$

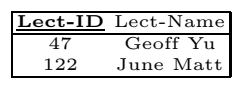

\section{MML Interpretation of Normalization}

Our simple example of the normalization process from the previous section has (ultimately) resulted in four distinct tables - namely, Student (table 5), Lecturer (table 6), Unit (table 3), and Stu-Unit-Rec (table 4). Normalization is nothing but judicious re-structuring of information via tables.

We now flesh out ideas from [9, footnote 187], [12, pp454-455] and [10, sec. 7.6]. In an MML or information-theoretic version of normalization, we can think of tables as a certain model (or hypothesis). Following equation (2), we can write the first-part message length (encoding the model) as:

$$
\# H=|<T>|+|<A>|+\sum_{t=1}^{T} A P_{t}
$$

where $\mathrm{T}$ is the number of tables, $\mathrm{A}$ is the number of attributes, $\langle T\rangle$ is an encoding of $T,\langle A\rangle$ is an encoding of $A,|\langle T\rangle|$ is the length of encoding $T$ and $|\langle A\rangle|$ is the length of encoding $A$. $A P_{t}$ denotes the encoding length of table $t$ 's attributes and its primary key. It is defined in equation (4) as:

$$
A P_{t}=\log _{2}(\mathrm{~A})+\log _{2}\left(\begin{array}{c}
A \\
a_{t}
\end{array}\right)+\log _{2}\left(\mathrm{a}_{t}\right)+\log _{2}\left(\begin{array}{c}
a_{t} \\
p_{t}
\end{array}\right)
$$

where $a_{t}$ is the number of attributes in the $t^{t h}$ table, $p_{t}$ denotes the number of attributes in the primary key. (We know that $1 \leq a_{t} \leq A$, so $\log _{2}(A)$ is the cost of encoding $a_{t}$, and $\log _{2}\left(\begin{array}{c}A \\ a_{t}\end{array}\right)$ is the cost of saying which particular $a_{t}$ attributes are in the $t^{\text {th }}$ table. Similarly, since $1 \leq p_{t} \leq a_{t}, \log _{2} a_{t}$ is the cost of encoding $p_{t}$, and $\log _{2}\left(\begin{array}{l}a_{t} \\ p_{t}\end{array}\right)$ is the cost of saying which particular $p_{t}$ attributes are in the primary key of the $t^{\text {th }}$ table.) Note that this is only one way of specifying the model. We have taken only the number of tables, attributes in each table and attributes constituting the PK in each table into account in specifying a model. Other models could be used. Note that the foreign keys (FKs) are not specified in this model - as the model encompasses information about the attributes in each table along with primary keys (PKs), the FKs can be found out by tracking the PK attribute of one table appearing in another table. For the sake of simplicity, we will not consider the effect(s) of $|\langle T\rangle|$ and $|\langle A\rangle|$ in the following discussion, as $|\langle A\rangle|$ appears in the encoding of each normalized form. We could (and implicitly do) assume a uniform prior on $|\langle T\rangle|$, but we could equally well instead have used (e.g.) a unary code $\left(\operatorname{Pr}(T)=2^{-T},|<T\rangle \mid=T\right)$ or the very slowly growing Wallace tree code [7, fig. 2.13 and sec. 2.1.14]. Hence, neglecting (near-)constant terms, we can (re-)write equation (3) as: 


$$
\# H=\sum_{t=1}^{T} A P_{t}
$$

In the following discussion we will assume that there are $m_{1}, m_{2}, m_{3}, m_{4}$, $m_{5}, m_{6}, m_{7}, m_{8}, m_{9}$ and $m_{10}$ unique instances of Stud-ID, Stud-Name, StudAddress, Stud-Course, Unit-No, Unit-Name, Lect-No, Lect-Name, Yr-Sem and Grade respectively (refer to table [7).

Table 7. Number of unique instances for each attribute in table 1, 1NF of our initial example

\begin{tabular}{|c|c|c|c|c|c|c|c|c|c|}
\hline Stud-ID & Stud-Name & Stud-Address & Stud-Course & Unit-No & Unit-Name & Lect-No & Lect-Name & Yr-Sem & Grade \\
\hline$m_{1}$ & $m_{2}$ & $m_{3}$ & $m_{4}$ & $m_{5}$ & $m_{6}$ & $m_{7}$ & $m_{8}$ & $m_{9}$ & $m_{10}$ \\
\hline$\overline{5}$ & 5 & $\overline{5}$ & 5 & 4 & $\overline{4}$ & $\overline{2}$ & $\overline{2}$ & 3 & 3 \\
\hline
\end{tabular}

The number of rows in the $1 \mathrm{NF}$ form of the table is an important variable. We have denoted it by $L$ in the preceding equations. $L=11$ in table 1 and depends on how many students are taking how many courses in each semester. We will later show that there is not a huge need for normalization if each student is taking only one unit, as $2 \mathrm{NF}$ will encode the same (amount of) information as $1 \mathrm{NF}$. As more students take more courses, the need for normalization arises.

Let us consider data in the $1 \mathrm{NF}$ Student table in table 1. We can write the $1 \mathrm{NF}$ encoding length $\left(I_{1 N F}\right)$ as the sum of the length of model $\left(H_{1 N F}\right)$ and length of data $\left(A_{1 N F}\right)$ encoded by this model as follows:

$$
\begin{aligned}
I_{1 N F} & =\# H_{1 N F}+\# A_{1 N F} \\
& =\# H_{1 N F}+L \times\left(\log _{2} m_{1}+\log _{2} m_{2}+\log _{2} m_{3}+\ldots \ldots+\log _{2} m_{10}\right)
\end{aligned}
$$

$\# H_{1 N F}$ in the preceding equation (equation (6) ) can be computed from equations (4) and (5). As there is only one table, $T=1$. There are 10 attributes $(A=10)$ and 3 attributes in the primary key $(p=3)$.

Consider the three tables used here in $2 \mathrm{NF}$ - i.e., Student, Unit and Stu-UnitRec (shown in tables 2, 3, 4). We can write the $2 \mathrm{NF}$ encoding length $\left(I_{2 N F}\right.$ ) as the sum of the length of model $\left(H_{2 N F}\right)$ and length of data $\left(A_{2 N F}\right)$ encoded by this model. Examining the 3 tables and their attributes, this gives:

$$
\begin{aligned}
I_{2 N F}= & \# H_{2 N F}+\# A_{2 N F} \\
= & \# H_{2 N F}+m_{1} \times\left(\log _{2} m_{1}+\log _{2} m_{2}+\log _{2} m_{3}+\log _{2} m_{4}+\log _{2} m_{7}+\log _{2} m_{8}\right) \\
& +m_{5} \times\left(\log _{2} m_{5}+\log _{2} m_{6}\right) \\
& +L \times\left(\log _{2} m_{1}+\log _{2} m_{5}+\log _{2} m_{9}+\log _{2} m_{10}\right)
\end{aligned}
$$

Like $\# H_{1 N F}$ (from equation (6) $)$, \# $H_{2 N F}$ in the preceding equation (equation (71) ) can also be computed from equations (44) and (57). There are 10 attributes $(A=10)$ in total and $T=3$ tables. The Student table has 6 attributes $\left(a_{1}=6\right)$ and $1 \mathrm{PK}$ attribute $\left(p_{1}=1\right)$. Similarly, the Unit table has 2 attributes $\left(a_{2}=2\right)$ and $1 \mathrm{PK}$ attribute $\left(p_{2}=1\right)$. The Stu-Unit-Rec table has 4 attributes $\left(a_{3}=4\right)$ 
and $3 \mathrm{PK}$ attributes $\left(p_{3}=3\right)$. The $\# A_{2 N F}$ part in equation (7) is the sum of the encoding lengths of the data in these 3 tables. Note the multiplication factors $m_{1}, m_{5}$ and $L$ in the encoding term, since there are $m_{1}$ rows in the Student table, $m_{5}$ rows in the Unit table and $L$ rows in the Stu-Unit-Rec table.

Moving from $2 N F$ to $3 N F$, the Student table in $2 N F$ is split into Student (table 5) and Lecturer (table 6). We can write the $3 \mathrm{NF}$ encoding length $\left(I_{3 N F}\right)$ as the sum of the length of model $\left(H_{3 N F}\right)$ and length of data $\left(A_{3 N F}\right)$ encoded by this model, noting that we replace the cost of the student table (table 2) in $2 \mathrm{NF}$ from equation (7) with the costs of the new (and more concise) student table (table 5) and the lecturer table (table 6).

$$
\begin{aligned}
I_{3 N F}= & \# H_{3 N F}+\# A_{3 N F} \\
= & \# H_{3 N F}+m_{1} \times\left(\log _{2} m_{1}+\log _{2} m_{2}+\log _{2} m_{3}+\log _{2} m_{4}+\log _{2} m_{7}\right) \\
& +m_{7} \times\left(\log _{2} m_{7}+\log _{2} m_{8}\right) \\
& +m_{5} \times\left(\log _{2} m_{5}+\log _{2} m_{6}\right) \\
& +L \times\left(\log _{2} m_{1}+\log _{2} m_{5}+\log _{2} m_{9}+\log _{2} m_{10}\right)
\end{aligned}
$$

$\# H_{3 N F}$ can also be computed from equations (4) and (5). There are $A=10$ attributes and $T=4$ tables in $3 N F$. The Student table has 5 attributes $\left(a_{1}=5\right)$ and $1 \mathrm{PK}$ attribute $\left(p_{1}=1\right)$. Since the Unit and Stu-Unit-Rec tables are already in $3 N F$, we have $a_{2}=2, p_{2}=1, a_{3}=4$ and $p_{3}=3$ from the previous discussion. The Lecturer table has 2 attributes $\left(a_{4}=2\right)$ and 1 PK attribute $\left(p_{4}=1\right)$.

The encoding length of data along with the model for each NF for our initial small example (of only $L=11$ rows in 1NF) is shown in table 8 . As we have moved to higher NFs, we have made our model more complicated as depicted by the encoding length $(\# H)$, but the data in the second part of the message $(\# A)$ is encoded more efficiently and its length has vastly decreased. As can be seen from equations (6), (7) and (8), all encodings depend on the parameter $L$. We see an improvement of $2 \mathrm{NF}$ over $1 \mathrm{NF}$ even for $L=11$ rows in this small example.

Table 8. Code length (bits) of model and data for different NFs on small example

\begin{tabular}{|c|c|c|c|}
\hline & $\# H$ (first part's length) & $\# A$ (second part's length) & total message length \\
\hline $1 \mathrm{NF}$ & 10.22 & 203.03 & 213.25 \\
$2 \mathrm{NF}$ & 36.45 & 154.89 & 191.34 \\
$3 \mathrm{NF}$ & 46.26 & 153.84 & 200.10 \\
\hline
\end{tabular}

Due to space constraints we have not included a lot of data in table 1. In this particular example with $L=11$ rows in $1 \mathrm{NF}$, the total message length appears (slightly) higher for $3 \mathrm{NF}$ than $2 \mathrm{NF}$. This should not be surprising considering the amount of data we have. Let us note from equations (7) and (8) that

$$
I_{3 N F}-I_{2 N F}=\left(\# H_{3 N F}-\# H_{2 N F}\right)+m_{7} \log _{2} m_{7}+\left(m_{7}-m_{1}\right) \log _{2} m_{8}
$$


On the not unreasonable assumption that $m_{7}=m_{8}$, then $I_{3 N F}-I_{2 N F}=\left(\# H_{3 N F}-\# H_{2 N F}\right)+\left(2 m_{7}-m_{1}\right) \log _{2} m_{7}$.

Provided that $m_{1}>2 m_{7}$ then, as $m_{1}$ or $m_{7}$ increases, the term $\left(2 m_{7}-m_{1}\right) \log _{2} m_{7}$ will become increasingly negative, eventually becoming larger in magnitude than $\left(\# H_{3 N F}-\# H_{2 N F}\right.$ ), whereupon $I_{3 N F}$ will become less than $I_{2 N F}$, at which point MML will then forever after prefer $3 \mathrm{NF}$ to $2 \mathrm{NF}$. This comparison between $m_{1}$ and $2 m_{7}$ is because in going from $2 \mathrm{NF}$ to $3 \mathrm{NF}$ we are removing a column of $m_{1}$ entries in the 2NF Student table (table 2) and replacing it with a new 3NF table (table 6. Lecturer) of 2 columns and $m_{7}$ rows.

So, now let us suppose that we have a more realistic (but still quite small) example of $m_{1}=100$ students, $m_{5}=30$ units and each student is taking an average of 3 courses (note $L=300$ ), setting the number of lecturers equal to $m_{7}=15$. The encoding lengths are given in table 9 , which is also a cross-section of figure $1(\mathrm{~b})$.

Table 9. Encoding length (in bits) of model and data for different NFs, Number of Students $\left(m_{1}\right)=100$, Number of Units $\left(m_{5}\right)=30$, Number of Lecturers $\left(m_{7}\right)=15$, $L=300$

\begin{tabular}{|c|c|c|c|}
\hline & $\# H$ (first part's length) & $\# A$ (second part's length) & total message length \\
\hline $1 \mathrm{NF}$ & 10.22 & 14210 & 14220 \\
$2 \mathrm{NF}$ & 36.45 & 8150 & 8186 \\
$3 \mathrm{NF}$ & 46.26 & 7876 & 7922 \\
\hline
\end{tabular}

To illustrate this point graphically, in figure 1 we see the effect on encoding length by varying and increasing $L$ and the number of students $\left(m_{1}\right)$. If each student is only taking one unit $\left(m_{1}=m_{5}\right), 2 \mathrm{NF}$ will not be beneficial even if the number of students is increased from 10 to say 10000. This is depicted in figure $1(\mathrm{a})$. Because $L=m_{1}$ and there is insufficient data to infer the partial dependencies required for $2 \mathrm{NF}$, the original $1 \mathrm{NF}$ table is adequate for $2 \mathrm{NF}$. Indeed, enforcing the premature creation of (superfluous) tables (enforced 2NF) can understandably be seen to increase the message length. Despite this, the transitive dependencies of Stud-ID $\rightarrow$ Lect-No $\rightarrow$ Lect-Name $\left(\right.$ with $m_{1}>2 m_{7}$ ) result in message length improvements when we go to $3 \mathrm{NF}$. Taking the unnecessarily enforced $2 \mathrm{NF}$ is improved by then converting it to $3 \mathrm{NF}$. But best of all is to take the original $1 \mathrm{NF}$ table as our $2 \mathrm{NF}$ (as there is insufficient data to suggest otherwise) and then convert this to 3NF. Figure 1(a) bears out this analysis.

As can be seen from figure 1(b) (of which the total message length in table 9 is a special case with $m_{1}=100$ and $L=3 m_{1}=300$ ), normalization from $1 \mathrm{NF}$ to $2 \mathrm{NF}$ is really beneficial as students enrol in more than one unit. The emphatic message length benefits visible in figure $1(\mathrm{~b})$ in going from $1 \mathrm{NF}$ to $2 \mathrm{NF}$ are most probably to be expected, with the pronounced benefit of normalization being self-evident when the number of students (enrolling in more than one unit) is large. The transitive dependency that we observed in figure 1(a) applies again 


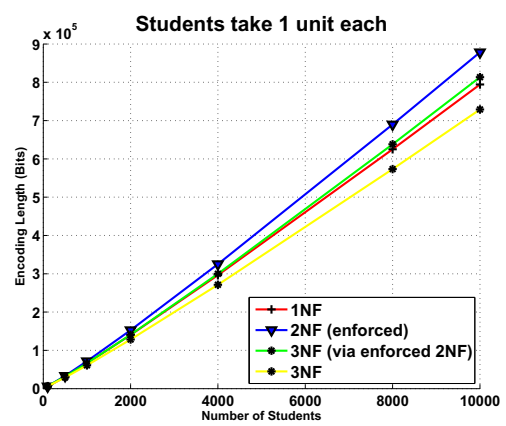

(a)

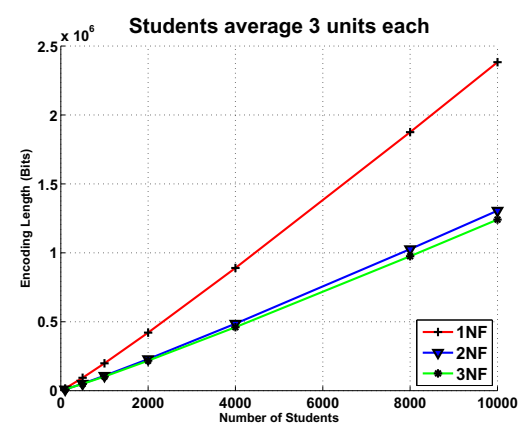

(b)

Fig. 1. Variation in total message length $(I)$ by varying number of students $\left(m_{1}\right)$ and $L$ for different NFs. The number of Units $\left(m_{5}\right)$ is set to 30 and the number of Lecturers $\left(m_{7}\right)$ is set to 15 . 1(a) $L=m_{1}, 1(\mathrm{~b}), L=3 m_{1}$.

here (with $m_{1} \geq 50>2 m_{7}=30$ ) as the number of students $\left(m_{1}\right)$ and their enrolments increases, whereupon MML again prefers the $3 \mathrm{NF}$ model.

\section{Conclusion and Future Work}

We have presented database normalization as a consequence of MML inference. With an example, we demonstrated a typical normalization procedure and analyzed the process using the MML framework. We found that with higher NFs, the model is likely to become more complicated, but the data encoding length is decreased. If there is a relationship or dependency in the data (according to database normalisation principles), then - given sufficient data - MML will find this. This suggests that normalization is - in some sense - simply following MML.

Though we have limited ourselves here to $1^{\text {st }}, 2^{\text {nd }}$ and $3^{\text {rd }}$ normal forms (NFs), applying MML can also be shown to lead to higher NFs such as Boyce-Codd Normal Form (BCNF), $4 \mathrm{NF}$ and 5NF. Indeed, recalling the notion of MML Bayesian network (see, e.g., 7, sec. 7.4][8] 9, sec. 0.2.5 and footnotes 53, 75 \& 218][10]), normalizing and breaking down tables into new tables can be thought of as a (MML) Bayesian net analysis - using the fact that (in some sense) databases could be said to have no noise. And, in similar manner, (the notion of) attribute inheritance (where different types of employee - such as pilot and engineer - have their own specific attributes as well as inheriting common employee attributes) can also be inferred using MML. General statistical consistency results (see, e.g., [8, sec. 11.3] 9, secs. 0.2.3 - 0.3.1][12, pp436-437][10, sec. 5.2]) appear to guarantee that - given sufficiently large data-sets and sufficient search time - MML will converge upon the data generation process, whatever it is, whatever the appropriate (possibly previously unseen) normalization (or regularities). Our initial results here provide clear supporting evidence of this claim. 


\section{References}

1. Codd, E.: A relational model of data for large shared data banks. Communications of the ACM (1979)

2. Date, C.: An Introduction to Database Systems. Addison-Wesley Longman, Amsterdam (1999)

3. William, K.: A simple guide to five normal forms in relational database theory. Communications of the ACM (1983)

4. Wallace, C.S., Boulton, D.M.: An information measure for classification. Computer Journal 11, 185-194 (1968)

5. Wallace, C.S., Freeman, P.R.: Estimation and inference by compact coding. Journal of the Royal Statistical Society series B 49(3), 240-252 (1987)

6. Wallace, C.S., Dowe, D.L.: Minimum message length and Kolmogorov complexity. Computer Journal 42(4), 270-283 (1999)

7. Wallace, C.S.: Statistical and Inductive Inference by Minimum Message Length. Information Science and Statistics. Springer, Heidelberg (May 2005)

8. Comley, J.W., Dowe, D.L.: Minimum message length and generalized Bayesian nets with asymmetric languages. In: Grünwald, P., Pitt, M.A., Myung, I.J. (eds.) Advances in Minimum Description Length: Theory and Applications, pp. 265-294. M.I.T. Press, Cambridge (April 2005)

9. Dowe, D.L.: Foreword re C. S. Wallace. Computer Journal 51(5), 523-560 (2008); Christopher Stewart WALLACE (1933-2004) memorial special issue

10. Dowe, D.L.: MML, hybrid Bayesian network graphical models, statistical consistency, invariance and uniqueness. In: Wood, J., Forster, M.R., Bandyopadhyay, P. (eds.) Handbook of the Philosophy of Science - (HPS Volume 7) Philosophy of Statistics, vol. 7, pp. 861-942. Elsevier, Amsterdam (2010)

11. Wallace, C.S., Dowe, D.L.: MML clustering of multi-state, Poisson, von Mises circular and Gaussian distributions. Statistics and Computing 10, 73-83 (2000)

12. Dowe, D.L.: Minimum Message Length and statistically consistent invariant (objective?) Bayesian probabilistic inference - from (medical) "evidence". Social Epistemology 22(4), 433-460 (2008) 